ARTICLE

Received 23 Dec 2014 | Accepted 17 Mar 2015 | Published 24 Apr 2015

\title{
Impact of charge transport on current-voltage characteristics and power-conversion efficiency of organic solar cells
}

\author{
Uli Würfel ${ }^{1,2, \star}$, Dieter Neher ${ }^{3, \star}$, Annika Spies $^{1,2} \&$ Steve Albrecht ${ }^{3, \dagger}$
}

This work elucidates the impact of charge transport on the photovoltaic properties of organic solar cells. Here we show that the analysis of current-voltage curves of organic solar cells under illumination with the Shockley equation results in values for ideality factor, photocurrent and parallel resistance, which lack physical meaning. Drift-diffusion simulations for a wide range of charge-carrier mobilities and illumination intensities reveal significant carrier accumulation caused by poor transport properties, which is not included in the Shockley equation. As a consequence, the separation of the quasi Fermi levels in the organic photoactive layer (internal voltage) differs substantially from the external voltage for almost all conditions. We present a new analytical model, which considers carrier transport explicitly. The model shows excellent agreement with full drift-diffusion simulations over a wide range of mobilities and illumination intensities, making it suitable for realistic efficiency predictions for organic solar cells.

\footnotetext{
${ }^{1}$ Department of Dye and Organic Solar Cells, Fraunhofer Institute for Solar Energy Systems ISE, Heidenhofstrasse 2, 79100 Freiburg, Germany. ${ }^{2}$ Material Research Centre FMF, University of Freiburg, 79104 Freiburg, Germany. ${ }^{3}$ Institute of Physics and Astronomy, University of Potsdam, 14476 Potsdam-Golm, Germany. ${ }^{*}$ These authors contributed equally to this work. † Present address: Helmholtz-Zentrum Berlin für Materialien und Energie GmbH, Institut für Silizium-Photovoltaik, Kekuléstrasse 5, 12489 Berlin, Germany. Correspondence and requests for materials should be addressed to U.W. (email: uli.wuerfel@ise.fraunhofer.de) or to D.N. (email: neher@uni-potsdam.de)
} 
W ith the development of new photoactive materials and the optimization of device architectures, the performance of organic solar cells has significantly increased over the last 5 years. However, their power-conversion efficiency (PCE) remains well below the Shockley-Queisser limit $^{1}$. This raises the question about the physical processes limiting the efficiency. In the past, several models have been put forward, mainly considering losses due to geminate recombination ${ }^{2,3}$, limiting the short-circuit current $J_{\mathrm{SC}}$, or due to non-radiative recombination pathways reducing mainly the fill factor (FF) and the open-circuit voltage $V_{\mathrm{OC}}$ (refs 4,5). Recent experimental and theoretical work, however, led to conclude that the performance of different polymer:fullerene blends to be largely affected by inefficient charge extraction due to low mobilities, in particular in systems with effective charge generation and/or large active layer thickness ${ }^{6-8}$.

To describe the current-voltage characteristics ( $J V$ curve) of a solar cell quantitatively by means of an analytical expression, researchers often use the Shockley equations ${ }^{4,9-16}$ :

$$
J(V)=J_{0}\left\{\exp \left[e V /\left(n_{\text {id }} k_{\mathrm{B}} T\right)\right]-1\right\}-J_{\text {gen }}
$$

with $J_{0}$ being the dark generation (or saturation) current density, $e$ the elementary charge, $V$ the potential difference between the contacts, $k_{\mathrm{B}}$ Boltzmann's constant and $T$ the temperature. In equation (1), the total current density $J$ is the sum of the current density of photogenerated charge carriers $J_{\text {gen }}$ and the recombination current density $J_{\mathrm{rec}}(V)=J_{0} \exp \left[e V /\left(n_{\mathrm{id}} k_{\mathrm{B}} T\right)\right]$. In the latter term, $n_{\text {id }}$ is the ideality factor, which depends on the exact recombination mechanism and hence on the reaction order. The recombination rate $R$ is a function of the density of (free) electrons and holes, $n_{\mathrm{e}}$ and $n_{\mathrm{h}}$, respectively, which themselves depend on the positions of the quasi Fermi levels. The quasi Fermi level of the electrons in the electron transport level (ETL, corresponding to the lowest unoccupied molecular orbital (LUMO) of the acceptor) is denoted as $E_{\mathrm{FE}}$ and the quasi Fermi level of the electrons in the hole transport level (HTL, corresponding to the highest occupied molecular orbital (HOMO) of the donor) is denoted as $E_{\mathrm{FH}}$. The recombination rate $R$ for a particular recombination process can generally be written as:

$$
R=k_{\mathrm{r}}\left(n_{\mathrm{e}} n_{\mathrm{h}}\right)^{\beta / 2}
$$

with $k_{\mathrm{r}}$ being the recombination coefficient and $\beta$ the reaction order of the recombination process. With equation (2), the recombination current density can be expressed as:

$$
\begin{aligned}
J_{\text {rec }} & =e k_{\mathrm{r}} \int_{0}^{d}\left(n_{\mathrm{e}} n_{\mathrm{h}}\right)^{\beta / 2} \mathrm{~d} x \\
& =e k_{\mathrm{r}} n_{\mathrm{i}}^{\beta} \int_{0}^{d}\left(\exp \left[\left(E_{\mathrm{FE}}-E_{\mathrm{FH}}\right) / k_{\mathrm{B}} T\right]\right)^{\beta / 2} \mathrm{~d} x
\end{aligned}
$$

where $n_{\mathrm{i}}$ is the intrinsic charge-carrier density, $d$ the thickness of the photoactive layer and $J_{0}=e k_{\mathrm{r}} n_{\mathrm{i}}^{\beta} d$ is the corresponding darkgeneration current density.

For infinitely large electron and hole conductivities, the quasi Fermi levels (and with that the quasi Fermi level splitting) are constant throughout the active layer. In addition, for properly chosen contacts, $E_{\mathrm{FE}}\left(E_{\mathrm{FH}}\right)$ aligns with the Fermi level of the electron (hole) contact, resulting in:

$$
E_{\mathrm{FE}}(x)-E_{\mathrm{FH}}(x)=e V
$$

at any position $x$ within the photoactive layer. In this case, equation (3) becomes $J_{\mathrm{rec}}=J_{0} \exp \left[\beta\left(E_{\mathrm{FE}}-E_{\mathrm{FH}}\right) / 2 k_{\mathrm{B}} T\right]$. The bias across the active layer can be further related to the applied voltage by $V=V_{\text {ext }}-R_{\mathrm{S}}$ J, with $R_{\mathrm{s}}$ being an external (constant) series resistance. Applying the assumption of infinite conductivities of equation (4) in equation (3) gives the Shockley equation (equation (1)) and a comparison with equation (3) delivers the correlation of the ideality factor with the reaction order:

$$
n_{\mathrm{id}}=2 / \beta
$$

For direct recombination, the rate is given by $R=k_{\mathrm{dir}} n_{\mathrm{e}} n_{\mathrm{h}}$, with $k_{\text {dir }}$ being the coefficient for direct recombination, and the ideality factor $n_{\text {id }}=1$ in this case. For Shockley-Read-Hall recombination $^{17}$, it is $n_{\mathrm{id}}=2$ and for Auger recombination ${ }^{18} n_{\mathrm{id}}=2 / 3$.

As transport was not considered in the derivation of equation (1), it can be regarded as an idealization that describes a device with infinitely large conductivities (and hence mobilities) of electrons and holes. When Shockley and co-workers ${ }^{9}$ derived their expression this was a reasonable assumption, as their aim was to describe the behaviour of diodes. By that time, diodes were solid-state devices comprising $p n$-junctions made from highly crystalline inorganic materials with rather high mobilities such as, for example, Ge, Si or GaAs.

In contrast to crystalline inorganic semiconductors, organic semiconductors display quite low mobilities ${ }^{19,20}$. Hence, the basic assumption of equation (4) is not fulfilled for organic solar cells.

To investigate the effect of limited transport properties, numerical simulations were performed with the semiconductor device simulation tool TCAD Sentaurus from Synopsys Inc. ${ }^{21}$. The model is based on mimicking the photoactive layer by a onedimensional effective semiconductor with the ETL corresponding to the LUMO of the acceptor phase and the HTL to the HOMO of the donor phase ${ }^{22}$. The electrodes are defined by their work functions and the surface recombination velocities of electrons and holes. As state-of-the-art organic solar cells employ thin interlayers from, for example, $\mathrm{TiO}_{\mathrm{x}}, \mathrm{ZnO}, \mathrm{MoO}_{3}, \mathrm{NiO}$ and $\mathrm{V}_{2} \mathrm{O}_{5}$ to enhance charge-carrier selectivity of the contacts ${ }^{23-26}$, electron- and hole-selective layers were included into the onedimensional stack. For the electron-selective layer the ETL is aligned to the ETL of the photoactive layer, whereas the holes encounter a large energetic barrier of $1.7 \mathrm{eV}$ and vice versa for the hole-selective layer (Supplementary Fig. 1), thus suppressing surface recombination at the electrodes. The parameters are summarized in Table 1 and realistic values were used ${ }^{27,28}$. Intragap states were not considered; only direct (bimolecular) recombination was taken into account according to $R=k_{\mathrm{dir}} n_{\mathrm{e}} n_{\mathrm{h}}$.

For sake of simplicity, the generation rate was restricted to the photoactive layer and assumed to be spatially homogeneous. Only balanced mobilities $\left(\mu_{\mathrm{e}}=\mu_{\mathrm{h}}=\mu\right)$ were considered and series or parallel resistances were not regarded in the simulations $\left(R_{\mathrm{S}}=1 / R_{\mathrm{P}} \equiv 0\right)$. Here we analyse the effect of charge-carrier transport on the current-voltage characteristics and PCE of single-junction photovoltaic devices. We demonstrate that it is meaningless to apply the Shockley equation to current-voltage curves under illumination and to extract information on physical parameters such as the recombination order or the apparent shunt resistance $R_{\mathrm{p}}$. Further, we propose a new analytical approach, which is suited to accurately reproduce simulated current-voltage characteristics for a wide range of parameters and thus to predict achievable PCEs.

\section{Results}

Drift-diffusion simulations of $J V$ curves. The simulated $J V$ curves under an intensity of ' 1 sun' are plotted in Fig. 1a as thin lines with symbols. The thick solid lines are results of our analytical model, which will be explained in detail further below.

This figure illustrates the large effect of charge-carrier mobility on the shape of the $J V$ curve under illumination. Lowering the 


\section{Table 1 | Parameters used for the numerical simulations.}

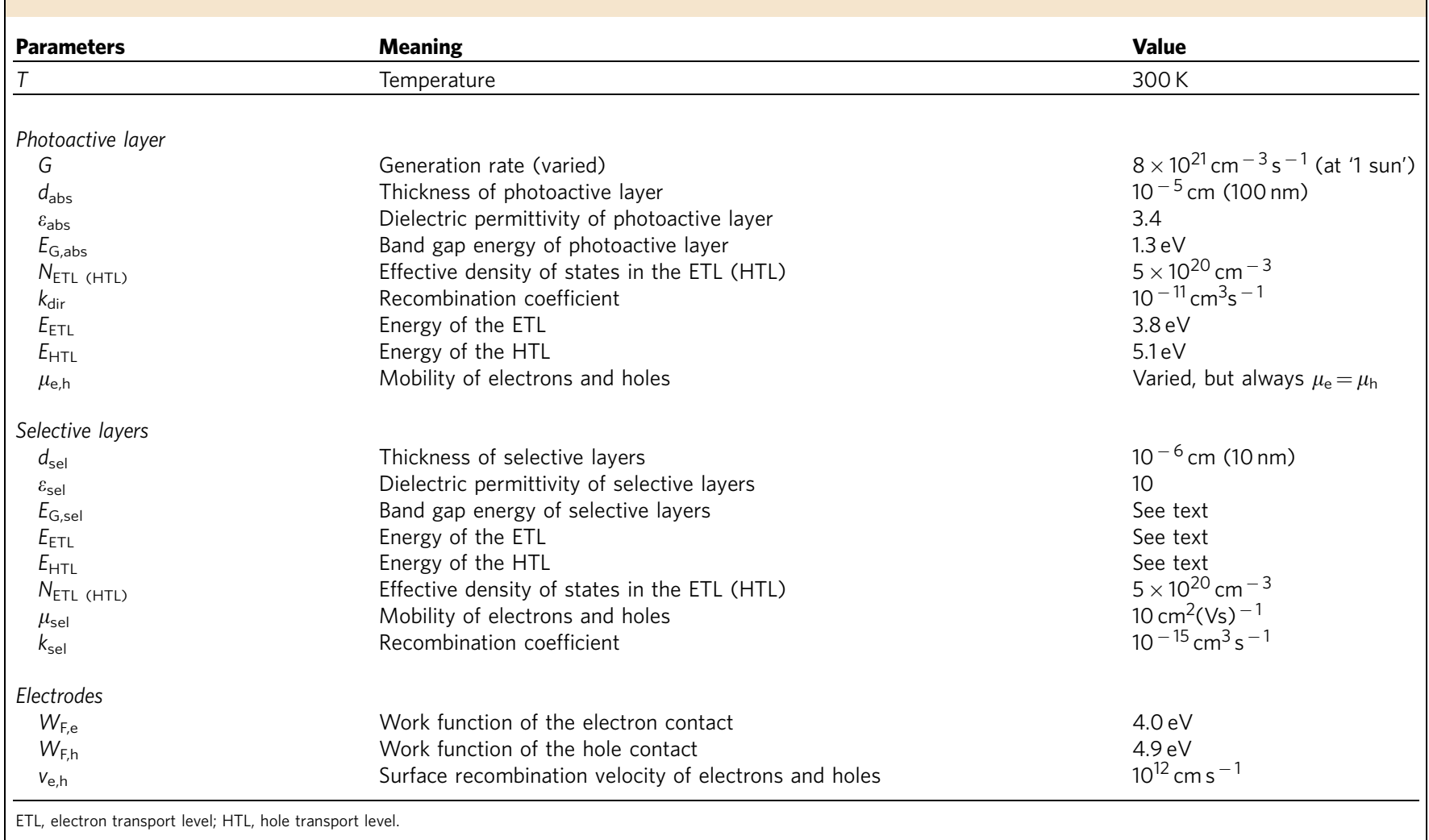
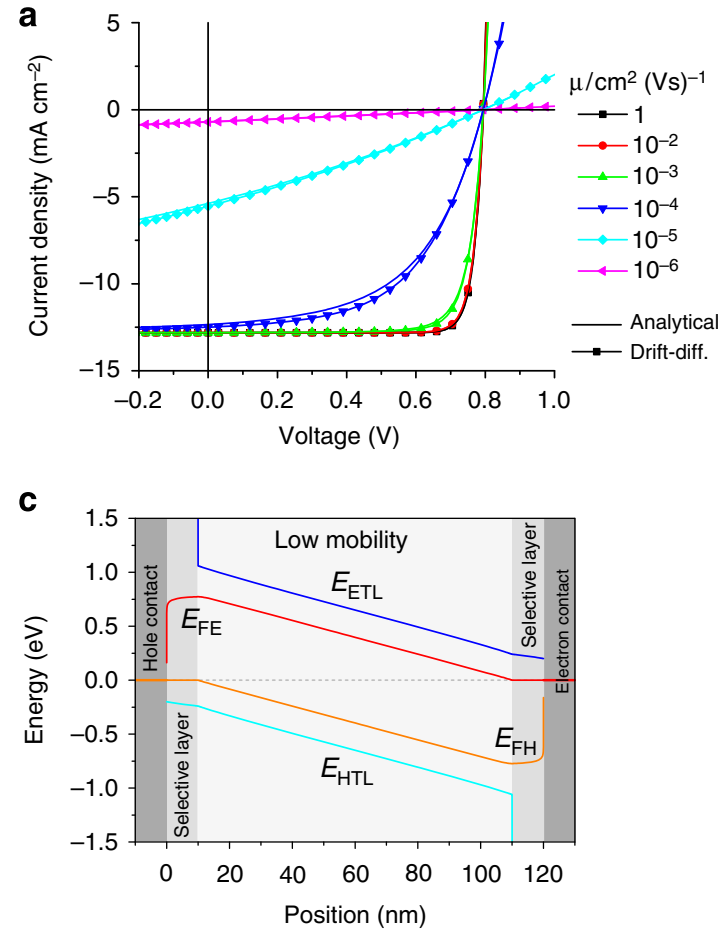

b
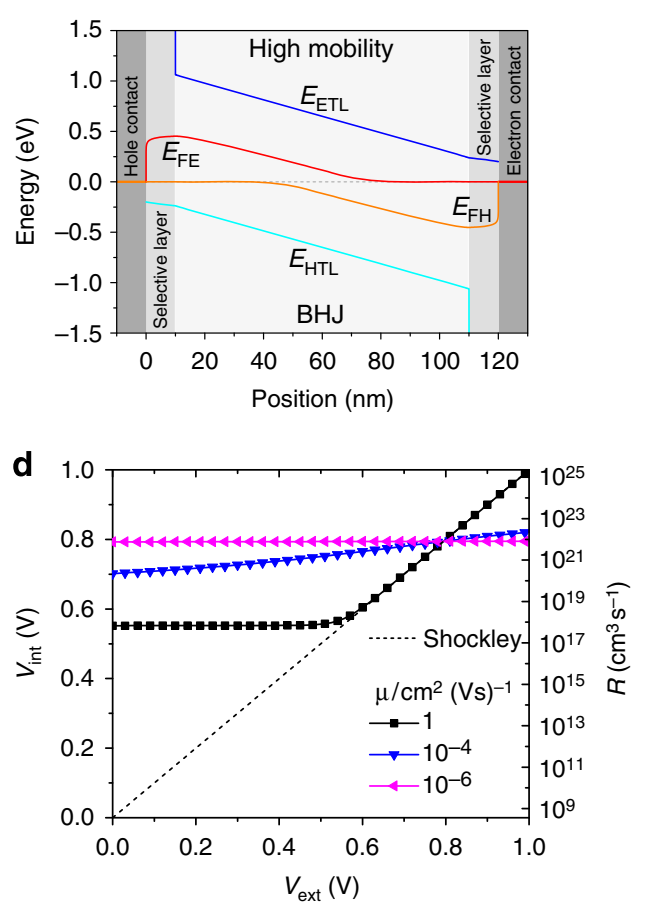

Figure 1 | Effect of the charge-carrier mobility on the $\mathbf{J V}$ characteristics. (a) JV curves for six different charge-carrier mobilities. Thin lines with symbols show drift-diffusion simulations with the parameters in Table 1, while thick lines are the results of our analytical approximation. (b,c) show the corresponding energy diagrams under short-circuit conditions $\left(V_{\text {ext }}=0 \mathrm{~V}\right)$ for $\mu=1$ and $10^{-6} \mathrm{~cm}^{2}\left(V_{s}\right)^{-1}$, respectively. (d) Internal voltage $V_{\text {int }}$ (left $y$ axis) versus external voltage $V_{\text {ext }}$ as extracted from the simulated $J V$ curves with equation (6) for three different mobilities, compared with the Shockley equation (dotted line). The right $y$ axis shows the corresponding recombination rate $R$. All graphs correspond to an illumination intensity of ' 1 sun'. 
mobilities first affects the FF, but $J_{\mathrm{SC}}$ is also significantly reduced for $\mu \leq 10^{-5} \mathrm{~cm}^{2}(\mathrm{Vs})^{-1}$. In addition, the forward current density decreases strongly with lower mobilities.

These changes go along with a drastic violation of the main condition in equation (4). Figure $1 \mathrm{~b}, \mathrm{c}$ plot calculated band diagrams for two different mobilities under ' 1 sun' illumination at $0 \mathrm{~V}$ (short-circuit conditions). If the conductivities were infinitely large, no separation of the quasi Fermi levels would occur in the active layer, which is indicated by the dashed horizontal lines, hence corresponding to the assumption of the Shockley equation. In contrast, even for a high-mobility $\mu_{\mathrm{e}}=$ $\mu_{\mathrm{h}}=1 \mathrm{~cm}^{2}(\mathrm{Vs})^{-1}$, the quasi Fermi level splitting $E_{\mathrm{FE}}-E_{\mathrm{FH}}$ in the bulk is considerably large and increases progressively with decreasing mobility. This proves that for all mobilities considered here, the quasi-Fermi level splitting is significantly larger than the external voltage $V_{\text {ext }}$ in the voltage range $0 \leq V_{\text {ext }} \leq V_{\text {OC. }}$. This has been confirmed recently by Schiefer et al. ${ }^{6,7}$ and Albrecht et al. ${ }^{8}$. The large splitting of the quasi-Fermi levels is the consequence of the accumulation of free charge carriers due to poor transport properties. As a result, non-geminate recombination is increased. This is the main cause for the continuous reduction of the FF and the short-circuit current density.

To identify a useful parameter describing the strength of recombination, we first calculated $J_{\text {rec }}$ from $J_{\text {rec }}(V)=J(V)+J_{\text {gen }}$. Next, an internal voltage $V_{\text {int }}$ is defined according to:

$$
e V_{\text {int }}=k_{\mathrm{B}} T \ln \frac{J_{\text {rec }}}{J_{0}}
$$

Here, $e V_{\text {int }}$ is the quasi-Fermi level splitting, which, if being constant throughout the entire active layer, would cause the same $J_{\text {rec }}$. Figure 1d shows $V_{\text {int }}$ as a function of the external voltage $V_{\text {ext }}$ under ' 1 sun' illumination for the Shockley equation (where $\left.V_{\text {int }} \equiv V_{\text {ext }}\right)$ and exemplarily for three different mobilities. For almost all conditions, $V_{\text {int }}$ is significantly larger than the external bias. Therefore, an important conclusion is that for typical mobilities in organic semiconductors, the Shockley equation massively underestimates the quasi-Fermi level spitting in the bulk and thereby the carrier density. High carrier densities have been reported by several authors even at short-circuit conditions, supporting the above findings ${ }^{29,30}$. For example, for the lowest mobility $\mu=10^{-6} \mathrm{~cm}^{2}(\mathrm{Vs})^{-1}$ and at $V_{\text {ext }}=0$, the device is internally almost under open-circuit conditions, causing $\sim 95 \%$ of the photogenerated carriers to recombine. Even for $\mu=1 \mathrm{~cm}^{2}(\mathrm{Vs})^{-1}$, the internal voltage $V_{\text {int }}$ has a considerably high value of $552 \mathrm{mV}$ under external short-circuit conditions. The above described effects are even more pronounced for increased thickness of the photoactive layer as well as for higher illumination intensities as can be seen in Supplementary Fig. 2a-e.

Based on the presented results, our simulations provide consistent proof that the condition of equation (4) is strongly violated in organic solar cells (except near OC conditions). Consequently, it is not meaningful to write the recombination current as a sole function of the bias across the active layer. We arrive at the conclusion that the Shockley equation is not applicable to organic solar cells, and that it cannot be used to analyse the current-voltage characteristics of these cells under illumination, to extract information about the recombination mechanism. This has several severe consequences. First, the difference between the current density under illumination, $J_{\text {illu, }}$, and in the dark, $J_{\text {dark, }}$ often denoted as the photocurrent $J_{\mathrm{ph}}$, has no physical meaning. It is neither equal to the overall photogenerated current nor does it mirror the recombination of only photogenerated charge. It is only $J_{\text {illu, }}$ which takes into account all generation processes and all recombination channels (photogenerated charge with photogenerated charge, injected with injected and photogenerated with injected). However, owing to the need of a driving force to establish $J_{\text {illu }}$ within the low-mobility semiconductor bulk, the analysis of $J_{\text {illu }}(\mathrm{V})$ does not deliver information about the true ideality factor (except near $V_{\mathrm{OC}}$ ). In addition, the widely used extended version of the Shockley equation: $J(V)=J_{0}\left(\exp \left[e\left(V-J R_{\mathrm{S}}\right) /\left(n_{\mathrm{id}} k_{\mathrm{B}} T\right)\right]-1\right)-J_{\mathrm{SC}}+\frac{V-J R_{\mathrm{S}}}{R_{\mathrm{P}}}$ is not applicable and the often-used approach to extract the shunt resistance $R_{\mathrm{p}}$ from the slope of $J_{\text {illu }}$ near short circuit is not useful. Finally, the PCE becomes not only a function of the bandgap but also of the mobility, even if the generation of charge carriers is field independent and non-geminate recombination is slow. These three issues will be considered in more detail in the following.

Generation current and photocurrent. As pointed out above, it is quite common to define 'the' photocurrent, $J_{\mathrm{ph}}$ via $J_{\mathrm{ph}}=J_{\text {illu }}-J_{\text {dark. }}$ Figure 2a plots this quantity, normalized by the generation current: $\left(J_{\text {illu }}-J_{\text {dark }}\right) / J_{\text {gen }}$, that is, the relative extraction efficiency of the photogenerated current $J_{\text {gen }}$ (which for an illumination intensity of ' 1 sun' was set to $J_{\text {gen }}=e \int_{0}^{d} G(x) \mathrm{d} x=12.82 \mathrm{~mA} \mathrm{~cm}^{-2}$ ). As expected, providing additional driving force by applying a negative bias voltage helps to extract more photogenerated charge carriers in those cases where the extraction at $V_{\text {ext }}=0$ is incomplete due to a low mobility. However, the quantity $J_{\text {illu }}-J_{\text {dark }}$ can by no means be interpreted as the generation current, that is, $J_{\text {gen. }}$. This is only true for mobilities being so large that the driving forces for the transport of electrons and holes can be neglected (and if external resistances can be neglected ${ }^{31}$ ). In fact, the extraction efficiency decreases strongly with increasing forward bias and the larger the mobilities the higher the voltage where the extraction efficiency starts to decrease. It should be noted here that for an ideal diode obeying equation (1) the difference between current under illumination and current in the dark is independent of voltage and equals the generation current: $J_{\text {illu }}-J_{\text {dark }} \equiv-J_{\text {gen }}$. The reason for the voltage dependence in forward direction is the fact that the concentration of electrons and holes for a given voltage $V_{\text {ext }}$ is higher for an illuminated solar cell than for one in the dark. The additional generation of charge carriers by the light source increases their concentration and hence also their conductivity. As a consequence, a smaller part of the applied voltage $V_{\text {ext }}$ is required as driving force for the transport and hence the internal voltage $V_{\text {int }}$, and thus the (recombination) current is higher. This can lead (depending on the exact parameters) to the oftenobserved effect of intercepting dark and illuminated $J V$ curves, that is, the $J V$ curve under illumination 'overtakes' the one measured in the dark. In Fig. $2 \mathrm{a}$, this becomes visible by the negative values of $\left(J_{\text {illu }}-J_{\text {dark }}\right) / J_{\text {gen }}$.

To highlight this phenomena, the spatial average of the effective charge-carrier conductivity $\sigma_{\mathrm{av}, \text { eff }}=\frac{1}{d} \int_{0}^{d} \sqrt{\sigma_{\mathrm{e}}(x) \sigma_{\mathrm{h}}(x)} \mathrm{d} x$ is plotted versus the mobility at ' 1 sun' for short-circuit conditions in Fig. 2b. The conductivity of electrons and holes is given by: $\sigma_{\mathrm{e}, \mathrm{h}}=e \mu_{\mathrm{e}, \mathrm{h}} \times n_{\mathrm{e}, \mathrm{h}}$. For a given mobility, the conductivity increases strongly under illumination due to the additional generation of charge carriers. The larger conductivity then leads to larger currents in forward direction and results in the crossing of dark and illuminated $J V$ curves. As expected, the increase in conductivity under illumination and short-circuit conditions is less pronounced for higher mobilities.

Ideality factor and shunt resistance. According to the Shockley theory, for a homogeneous carrier distribution and with equation (4) being valid, the value of the ideality factor is exclusively determined by the recombination order. However, for 
a

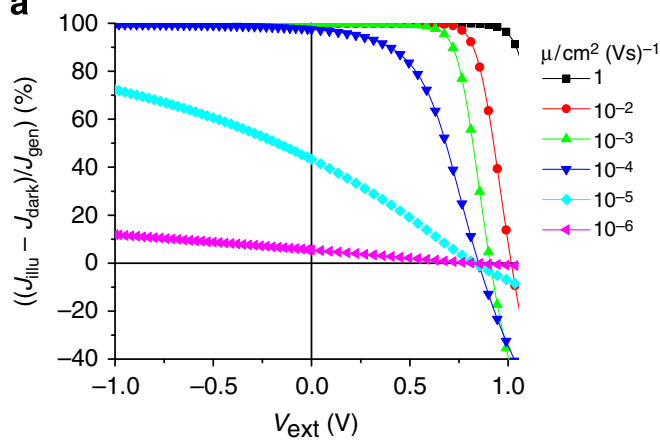

C

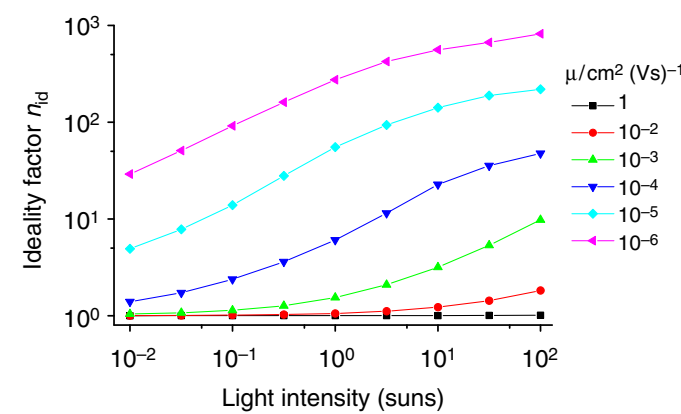

b

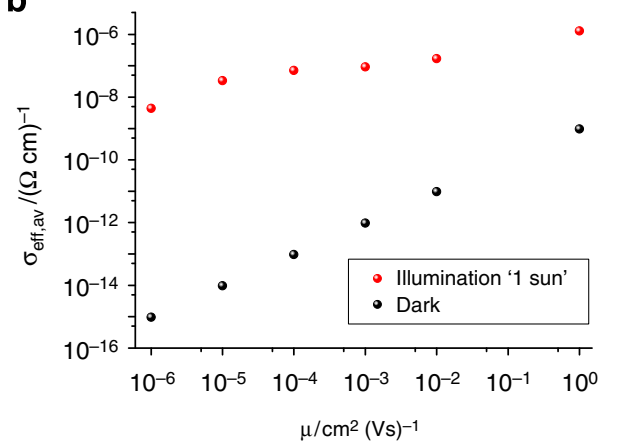

d

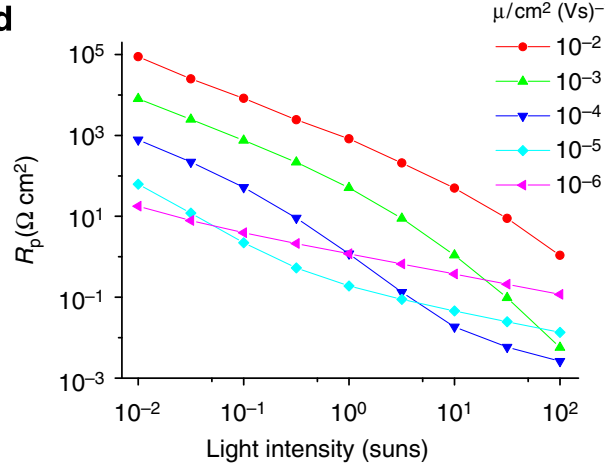

Figure 2 | Photoconductivity and ideality factor. (a) Relative extraction rate of the photogenerated current as a function of the external voltage for six different mobilities at an illumination intensity of ' 1 sun'. (b) Corresponding effective average conductivity (see text) at $V_{\text {ext }}=0$ as a function of mobility under illumination and in the dark. (c) Ideality factor $n_{\text {id }}$ as a function of light intensity for six different mobilities. (d) Apparent shunt resistance $R_{\mathrm{p}}$ as the function of light intensity as determined from the inverse slope of the simulated $J_{\text {illu }}(V)$ at $V=0$. The case of $\mu=1 \mathrm{~cm}^{2}(V \mathrm{~s}){ }^{-1}$ was left out for better visualization.

moderate or small mobilities the relative recombination rate and hence the ideality factor will become a function of both the mobility and the illumination intensity. To illustrate this effect, $J V$ curves were simulated for different mobilities and illumination intensities between 0.01 suns and 100 suns (see Supplementary Fig. 2 for exemplary curves). From these, the ideality factor was determined via:

$$
n_{\mathrm{id}}=\left(\frac{k_{\mathrm{B}} T}{e} \frac{\mathrm{d}}{\mathrm{d} V} \ln \left[J(V) / J_{0}\right]\right)^{-1}
$$

Hereby, a linear fit (of the logarithmically plotted curve) was performed in the fourth quadrant, that is, $0 \leq V \leq V_{\text {OC. }}$ The results are plotted in Fig. 2c, showing a very large variation of the ideality factor with charge-carrier mobility and illumination intensity. The only exception is $\mu=1 \mathrm{~cm}^{2}(\mathrm{Vs})^{-1}$ where the ideality factor is unity for all light intensities. The lower the value for $\mu$, the more the ideality factor deviates from 1 and the lower the intensity where $n_{\mathrm{id}}$ starts to increase. Notably, the apparent ideality factor determined via equation (7) can become very large, although direct free charge-carrier recombination was the only recombination pathway in our simulations and, therefore, $n_{\mathrm{id}}=1$ should be expected. This proves that applying the Shockley equation to the $J V$ curves of low-mobility carrier devices results in ideality factors that lack real physical meaning. As shown in Fig. 2c, this violation becomes most severe for large generation currents (efficient charge generation and/or high illumination intensity) and low mobilities.

It has, indeed, become quite common to deduce information on the order of recombination and with that on the value of the ideality factor from other approaches than fitting $J_{\text {illu }}(V)$ curves in the fourth quadrant. For example, the bias dependence of the dark current density or of the electroluminescence intensity is analysed to determine $n_{\mathrm{id}}{ }^{32,33}$. However, as shown in Supplementary Fig. 3, the applicability of this approach becomes questionable for low carrier mobilities and high currents. Alternatively, the analysis can be restricted to the exponential part of dark $J V$ curves, but this requires devices with very-low leakage currents ${ }^{34}$. A more accurate method is the determination of the ideality factor from the dependence of $V_{\mathrm{OC}}$ on the light intensity. Here, the charge-carrier densities vary over several orders of magnitude and thus more information can be extracted $^{35,36}$. Although these measurements are not influenced by carrier transport, the accurate analysis of the results becomes difficult if the spatial distributions of electrons and holes are strongly asymmetric, which is the case, for example, for a small layer thickness or unintentional background doping ${ }^{13,37,38}$. In addition, surface recombination will alter the carrier profiles ${ }^{26}$ and lead to a reduction of the ideality factor.

We also find that low mobilities cause an apparent shunt resistance $R_{\mathrm{p}}$ (although $1 / R_{\mathrm{P}}=0$ in all our simulations). The concept of 'the' shunt resistance is frequently used in the literature to account for the non-zero slope of the $J_{\text {illu }}(V)$ curve at short-circuit conditions: $R_{\mathrm{P}}=\left.(\mathrm{d} J(V) / \mathrm{d} V)^{-1}\right|_{V=0}$. The analysis of experimental $J V$ curves with this approach yields values for $R_{\mathrm{p}}$ of typically few tens to several thousand $\Omega \mathrm{cm}^{2}$ (refs 39-41). Our simulations reveal that non-zero slopes of the $J V$ curves are an inevitable consequence of low charge-carrier mobilities. Values for the apparent shunt resistance deduced from the simulated $J V$ curves vary, indeed, over a very wide range, depending strongly on mobility and generation rate (Fig. 2d). The large discrepancy between the value of $R_{\mathrm{p}}$ used in the simulation (infinity) and the one deduced from the slope of the simulated $J V$ curves with the extended Shockley equation again proves its non-applicability to organic solar cells for virtually any typical mobility and illumination intensity. 
An analytical model for $J V$ curves of organic solar cells. For non-zero currents, the voltage drops related to the transport of electrons and holes through a resistive medium, that is, the photoactive layer, results in large differences between the external voltage $V_{\text {ext }}$, which is applied (and which can be measured directly), and the internal voltage $V_{\text {int }}$. As only the latter determines the recombination rate, the description of a $J V$ curve with equation (1) using $V_{\text {ext }}$ becomes worse as the charge-carrier conductivities get lower, and any approach to relate $J_{\text {illu }}$ to $V_{\text {ext }}$ in a trivial form will fail.

However, if we recalculate $J V$ curves with the help of equation (1) but replace $V_{\text {ext }}$ with $V_{\text {int }}\left(V_{\text {ext }}\right)$, the resulting curves are identical to the ones that were simulated with different mobilities. This is of course expected, as we excluded all loss mechanisms other than direct recombination between electrons and holes.

An interesting question is therefore how this internal voltage can be determined for real devices. One approach was followed by Würfel et al. ${ }^{42,43}$ with dye solar cells, where they managed to incorporate a third electrode into a dye solar cell, thus contacting the electron transport material, that is, the nanocrystalline TiOx, on both sides. Thus, they could show directly that under external short-circuit conditions, the internal voltage was already $\sim 75 \%$ of the $V_{\text {OC }}$. This is in full accordance with the analysis presented here.

Although the approaches outlined above are not applicable to organic solar cells, the authors demonstrated recently two different methods to arrive at an approximate value for $V_{\text {int }}$ in a working device ${ }^{7,8}$. Both relied on the assumption that the gradients of the quasi Fermi energies are constant throughout the photoactive layer, and that they are identical for electrons and holes (equation (8)). Although the assumption is not strictly fulfilled in real solar cells, it seems to be a reasonable approach as can be seen from the simulation results shown in Fig. 1b,c. We will show in the following that under this approximation, an accurate analytical description of the $J V$ curves under illumination becomes feasible for a wide range of mobilities and generation currents. First, the gradient of the quasi Fermi potential is related to the current density $J_{e, h}$ and the electrical conductivity $\sigma_{\mathrm{e}, \mathrm{h}}$ of electrons and holes via ${ }^{7}$

$$
\begin{aligned}
\operatorname{grad} E_{\mathrm{F}, \mathrm{E}}(x) & =e \frac{J_{\mathrm{e}}(x)}{\sigma_{\mathrm{e}}(x)}=\operatorname{grad} E_{\mathrm{F}, \mathrm{H}}(x)=e \frac{J_{\mathrm{h}}(x)}{\sigma_{\mathrm{h}}(x)}=\text { const. } \\
& =\frac{e\left(V_{\mathrm{ext}}-V_{\mathrm{int}}\right)}{d}=\frac{e V_{\mathrm{tr}}}{d}
\end{aligned}
$$

Therein, $\sigma_{\mathrm{e}, \mathrm{h}}=e \mu_{\mathrm{e}, \mathrm{h}} \times n_{\mathrm{e}, \mathrm{h}}$, with $\mu_{\mathrm{e}, \mathrm{h}}$ being the charge-carrier mobilities. $V_{\text {tr }}$ is the voltage drop required for the charge-carrier transport through the active layer, which can also be expressed as $V_{\mathrm{tr}}=J R_{\mathrm{tr}}$. The transport resistance $R_{\mathrm{tr}}$ is a non-ohmic resistance related to the charge-carrier conductivities and the thickness of the photoactive layer. It follows from equation (8) that the internal voltage is also constant throughout the photoactive layer. In this case, the external voltage can be related to the internal voltage via

$$
V_{\mathrm{ext}}=V_{\mathrm{int}}+J R_{\mathrm{tr}}=V_{\mathrm{int}}+\frac{d}{\sigma_{\mathrm{e}}+\sigma_{\mathrm{h}}} J
$$

The conductivity can be further approximated by

$$
\sigma=\sigma_{\mathrm{e}}+\sigma_{\mathrm{h}}=2 e \mu_{\mathrm{eff}} n_{\mathrm{i}} \exp \left[e V_{\mathrm{int}} / 2 k_{\mathrm{B}} T\right]
$$

with $n_{\mathrm{i}}$ being the intrinsic carrier density and $\mu_{\mathrm{eff}}=\sqrt{\mu_{\mathrm{e}} \mu_{\mathrm{h}}}$ being an effective mobility (see Supplementary Note 1 for the derivation of these terms).
To analytically describe $J\left(V_{\text {ext }}\right)$, first the recombination current is calculated via

$$
\begin{aligned}
J_{\text {rec }}\left(V_{\text {int }}\right) & =e d k_{\mathrm{r}} n_{\mathrm{e}} n_{\mathrm{h}}=e d k_{\mathrm{r}} n_{\mathrm{i}}^{2} \exp \left[e V_{\text {int }} / k_{\mathrm{B}} T\right] \\
& =J_{0} \exp \left[e V_{\text {int }} / k_{\mathrm{B}} T\right]
\end{aligned}
$$

Next, the total current is obtained by adding the generated current:

$$
J\left(V_{\text {int }}\right)=J_{0}\left\{\exp \left[e V_{\text {int }} / k_{\mathrm{B}} T\right]-1\right\}-J_{\text {gen }}
$$

Finally, by using equations (12) and (10) for equation (9), we arrive at

$$
V_{\text {ext }}\left(V_{\text {int }}\right)=V_{\text {int }}+\frac{d}{2 e \mu_{\text {eff }} n_{\mathrm{i}} \exp \left[e V_{\text {int }} / 2 k_{\mathrm{B}} T\right]} J\left(V_{\text {int }}\right)
$$

By these means, the current density and the external voltage can both be calculated from $V_{\text {int }}$. As values for $\mu$ and $k_{\mathrm{r}}$ can however be determined separately ${ }^{44-48}$, the intrinsic charge-carrier density $n_{\mathrm{i}}$ remains as the only fitting parameter.

As a first test of the applicability of this approach, we have calculated $J V$ curves with the parameters given in Table 1 and compared them with the $J V$ curves from the full drift-diffusion simulations. We find excellent agreement between the simulated and the analytic approach for all mobilities as demonstrated in Fig. 1a.

We also find good agreement when applying this approach to a layer thickness of $300 \mathrm{~nm}$ and when changing the illumination intensity to either 0.1 suns or 10 suns (see Supplementary Fig. 2). Thus, for balanced mobilities our analytical approach shall provide an accurate analytical description of $J V$ curves of organic solar cells over a wide range of mobilities and generation currents. The case of unbalanced mobilities is shown in the Supplementary Fig. 4 . It can be seen that in this case our approach delivers very reasonable results as well.

The achievable PCE. Finally, we consider the effect of the charge-carrier mobility on the achievable PCE. Figure 3 shows the efficiency as a function of the absorber band gap for different mobilities, either with the analytical approach and with our drift-diffusion simulations, compared with the prediction by the model of Scharber et al. ${ }^{49}$. A variable offset between the LUMO levels of donor and acceptor was used, depicted in Fig. 3a. The band gap of the donor was used to calculate the photogenerated current via $J_{\text {gen, } \mathrm{SQ}}\left(E_{\mathrm{G}, \mathrm{abs}}\right)=$ $e \int_{E_{\mathrm{G}, \mathrm{abs}}}^{\infty} J_{\gamma, \mathrm{AM} 1.5 \mathrm{G}}(\hbar \omega) \mathrm{d} \hbar \omega$ assuming full absorption of all photons of the AM1.5G spectrum with photon energies $\hbar \omega \geq E_{\mathrm{G} \text {,abs, }}$, according to the Shockley-Queisser limit. The dark-generated current $J_{0, \mathrm{SQ}}\left(E_{\mathrm{G}, \mathrm{eff}}\right)$ was determined using the band gap of the effective semiconductor on the basis of the black body spectrum with $\quad T=300 \mathrm{~K}: \quad J_{0, \mathrm{SQ}}\left(E_{\mathrm{G}, \text { eff }}\right)=\frac{e}{4 \pi^{2} \hbar^{3} c} \int_{E_{\mathrm{G}, \text { eff }}}^{\infty} \frac{(\hbar \omega)^{2}}{\exp \left[\hbar \omega / k_{\mathrm{B}} T\right]-1} \mathrm{~d} \hbar \omega$, where $c$ is the speed of light. This way, we made use of the fact that the absorption via charge transfer (states) does hardly contribute to the overall photogenerated current, while luminescence measurements show that most charge carriers do recombine via the effective semiconductor gap ${ }^{4,50,51}$. It is noteworthy that our study focuses on the influence of limited charge-carrier transport and the general findings presented here would not be altered even if the absorption via the effective band gap played a more significant role.

Next, an amplification factor $\alpha$ was determined as the ratio of $J_{0}$ (using the parameters in Table 1) and $J_{0, \mathrm{SQ}}: \alpha J_{0, \mathrm{SQ}}\left(E_{\mathrm{G}, \text { eff }}\right)=J_{0}\left(E_{\mathrm{G}, \text { eff }}\right)=e k_{\mathrm{r}} n_{\mathrm{i}}{ }^{\beta} d \rightarrow \alpha=5564$. This amplification factor expresses the fact that in organic solar cells, most charge carriers do not recombine radiatively (in contrast to the Shockley-Queisser limit), but rather through additional, non- 
a

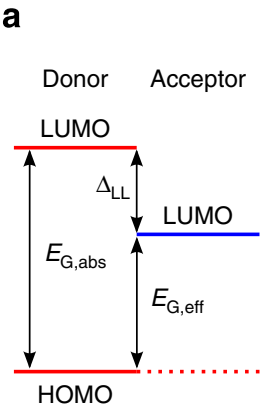

HOMO b

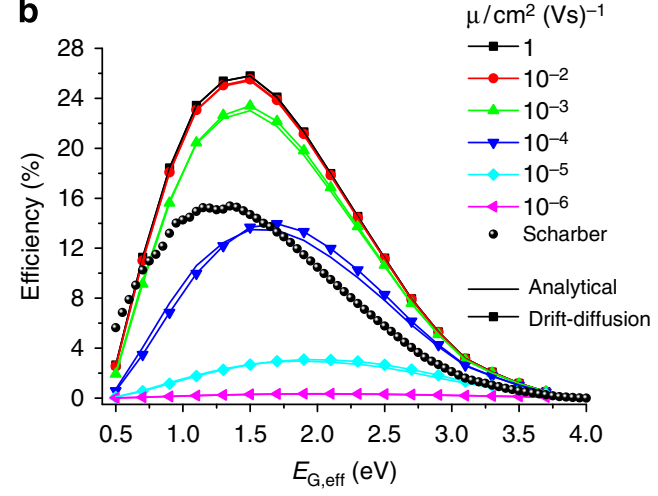

c

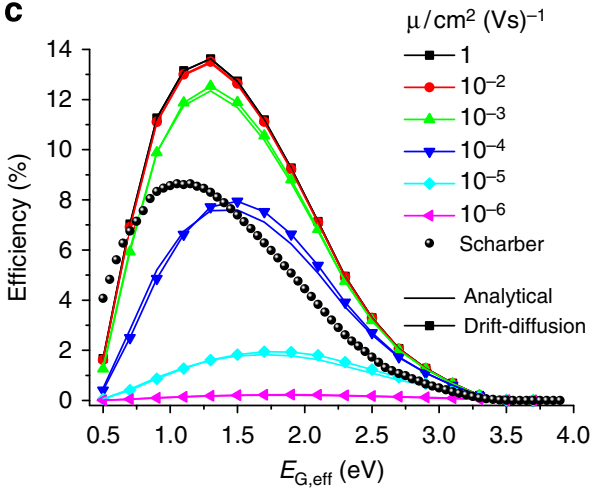

Figure 3 | The effect of mobility on the achievable PCE. (a) Scheme of the two band gaps involved. (b) Efficiencies are plotted as a function of effective semiconductor band gap, without an offset between donor and acceptor, that is, $E_{\mathrm{G}, \mathrm{abs}}=E_{\mathrm{G} \text {,eff }}$ and (c) with an offset $\Delta_{\mathrm{LL}}$ of $0.5 \mathrm{eV}$. Shown are the results for six different mobilities calculated either via the analytical approach (thin lines with symbols) or via drift-diffusion simulations (thick lines) in comparison with the Scharber model ${ }^{49}$. For parameters, see text.

radiative pathways ${ }^{4,51}$. For simplicity, this $\alpha=5564$ was used to calculate the dark-generated current for each effective band gap according to $J_{0}\left(E_{\mathrm{G}, \mathrm{eff}}\right)=\alpha J_{0, \mathrm{SQ}}\left(E_{\mathrm{G}, \text { eff }}\right)$.

Figure $3 \mathrm{~b}$ shows the results for equal band gaps of absorber and effective semiconductor, that is, no offset $\Delta_{\mathrm{LL}}$ between the LUMO levels of donor and acceptor (and of course also between their HOMO levels for excitons generated in the acceptor), while Fig. $3 c$ corresponds to $\Delta_{\mathrm{LL}}=0.5 \mathrm{eV}$. The results of our analytical approach are well in accordance with the full drift-diffusion simulations, meaning that the assumptions leading to equation (13) remain valid for a wide range of bandgaps and mobilities. The figures also demonstrate the large impact of the charge-carrier mobility on the device efficiency. Efficiencies beyond $25 \%$ are predicted only for zero offset, a condition that is rather unlikely to realize with organic donor-acceptor blends. Nevertheless, efficiencies of $12 \%$ and higher are within reach for a realistic offset of $0.5 \mathrm{eV}$, provided that the mobility is in excess of $10^{-3} \mathrm{~cm}^{2}(\mathrm{Vs})^{-1}$.

These predictions are in good agreement to recent efficiency values. For example, Proctor et al. ${ }^{52}$ compared mobility and FF values for various solution processed small molecule-based bulk heterojunction solar cells. It is shown that balanced mobilities in excess of $2 \times 10^{-4} \mathrm{~cm}^{2}(\mathrm{Vs})^{-1}$ are needed to achieve high FFs (and PCEs). Notably, a record efficiency of $10.8 \%$ was achieved in blends of carefully designed polymers with fullerene ${ }^{53}$. These blends had exceptionally high hole mobilities of $1.5-3.0 \times 10^{-2} \mathrm{~cm}^{2}(\mathrm{Vs})^{-1}$. Given the fact that the external quantum efficiency (EQE) was about 0.85 on average and that $E_{\mathrm{G}, \mathrm{abs}}$ was about $1.55 \mathrm{eV}$, efficiencies of $>13 \%$ should be in reach on further optimization of the absorption properties, consistent with our predictions in Fig. 3.

For comparison, we plotted the values according to the model of Scharber et al. ${ }^{49}$ Scharber et al. predicted PCE for various values of $E_{\mathrm{G} \text {,abs }}$ and $E_{\mathrm{G}, \text { eff }}$, assuming a constant value of 0.65 for both $\mathrm{EQE}$ and $\mathrm{FF}$, and assuming $\mathrm{e} V_{\mathrm{OC}}=E_{\mathrm{G}, \mathrm{eff}}-0.3 \mathrm{eV}$. The model of Dennler et al. ${ }^{54}$ is very similar, using a value of 0.9 for the EQE and 0.7 for the FF. As both models do not consider charge-carrier transport, they are not capable to predict efficiencies as a function of mobility. A more sophisticated model was presented by Koster et al. ${ }^{2}$ The authors analysed the effect of CT absorption, reorganization energy and dielectric permittivity $\varepsilon$ on the device performance, considering both geminate and non-geminate recombination. An important result of this work is that raising $\varepsilon$ allows for smaller offsets $\Delta_{\mathrm{LL}}$, enabling efficiencies beyond $20 \%$. However, the effect of charge-carrier mobility is not explicitly treated in this work.

As there is no fundamental reason for $\Delta_{\mathrm{LL}}$ to be at least $0.5 \mathrm{eV}$, we also determined the maximum efficiency for smaller offsets. For mobilities of $10^{-2} \mathrm{~cm}^{2}(\mathrm{Vs})^{-1}$ or larger and $\Delta_{\mathrm{LL}}=0.3 \mathrm{eV}$, we find an efficiency of $18 \%$, and for $\Delta_{\mathrm{LL}}=0.2 \mathrm{eV}$ the maximum efficiency is already slightly above $20 \%$. If the recombination coefficient $k_{\mathrm{r}}$ could be reduced by one order of magnitude, the $V_{\text {OC }}$ would increase by $59.6 \mathrm{mV}$ (at $300 \mathrm{~K}$ ). As the FF is also improved, the maximum efficiency for $\Delta_{\mathrm{LL}}=0.2 \mathrm{eV}$ reaches $22 \%$ for mobilities of at least $10^{-2} \mathrm{~cm}^{2}(\mathrm{Vs})^{-1}$ and even for mobilities of $10^{-4} \mathrm{~cm}^{2}(\mathrm{Vs})^{-1}$, it still is almost $17 \%$.

\section{Discussion}

We demonstrated that the Shockley equation cannot be applied to low-mobility materials such as those typically used in organic solar cells. The poor transport properties cause accumulation of charge carriers in the photoactive layer and this effect becomes more and more prominent with decreasing mobility. As a consequence, for almost all conditions encountered in organic solar cells, the separation of the quasi Fermi levels in the photoactive layer (internal voltage) differs substantially from the externally applied voltage. For this reason, there is no trivial relation between the charge-carrier concentrations in the illuminated solar cell and the external voltage, which is however an assumption of the Shockley equation. A further consequence is that parameters such as ideality factors or apparent shunt resistance determined via the simple or extended Shockley equation will result in values that lack real physical meaning. Therefore, it is not possible to extract correct information about the reaction order of the recombination process and the photogenerated current, by applying the Shockley equation to current-voltage characteristics of organic solar cells under illumination.

An analytical model is presented that explicitly considers the implication of poor charge-carrier transport. We have obtained excellent agreement of the presented analytical model with the results of full drift-diffusion simulations for a wide range of mobilities, illumination intensities and active layer thicknesses. In contrast to other models, it allows predicting efficiency potentials by explicit consideration of charge-carrier mobilities, which has been very tedious to date in an analytical way. 


\section{References}

1. Shockley, W. \& Queisser, H. J. Detailed balance limit of efficiency of p-n junction solar cells. J. Appl. Phys. 32, 510-519 (1961).

2. Koster, L. J. A., Shaheen, S. E. \& Hummelen, J. C. Pathways to a new efficiency regime for organic solar cells. Adv. Energy Mater. 2, 1246-1253 (2012).

3. Koster, L. J. A., Mihailetchi, V. D. \& Blom, P. W. M. Ultimate efficiency of polymer/fullerene bulk heterojunction solar cells. Appl. Phys. Lett. 88, 93511 (2006).

4. Vandewal, K., Tvingstedt, K., Gadisa, A., Inganäs, O. \& Manca, J. V. On the origin of the open-circuit voltage of polymer-fullerene solar cells. Nat. Mater. 8, 904-909 (2009).

5. Gruber, M. et al. Thermodynamic efficiency limit of molecular donor-acceptor solar cells and its application to diindenoperylene/C60-based planar heterojunction devices. Adv. Energy Mater. 2, 1100-1108 (2012).

6. Schiefer, S., Zimmermann, B., Glunz, S. W. \& Würfel, U. Applicability of the suns-voc method on organic solar cells. IEEE J. Photovoltaics 4, 271-277 (2014)

7. Schiefer, S., Zimmermann, B. \& Würfel, U. Determination of the intrinsic and the injection dependent charge carrier density in organic solar cells using the Suns-VOC method. J. Appl. Phys. 115, 44506 (2014).

8. Albrecht, S. et al. Quantifying charge extraction in organic solar cells: the case of fluorinated PCPDTBT. J. Phys. Chem. Lett. 5, 1131-1138 (2014).

9. Shockley, W. The Theory of $\mathrm{p}-\mathrm{n}$ junctions in semiconductors and $\mathrm{p}-\mathrm{n}$ junction transistors. Bell Syst. Tech. J. 28, 435-489 (1949).

10. Goucher, F., Pearson, G., Sparks, M., Teal, G. \& Shockley, W. Theory and experiment for a germanium p-n junction. Phys. Rev. 81, 637-638 (1951).

11. Chirvase, D. et al. Temperature dependent characteristics of poly $(3$ hexylthiophene)-fullerene based heterojunction organic solar cells. J. Appl. Phys. 93, 3376 (2003).

12. Foertig, A., Rauh, J., Dyakonov, V. \& Deibel, C. Shockley equation parameters of P3HT:PCBM solar cells determined by transient techniques. Phys. Rev. B 86, 115302 (2012)

13. Kirchartz, T. \& Nelson, J. Meaning of reaction orders in polymer:fullerene solar cells. Phys. Rev. B 86, 165201 (2012).

14. Schilinsky, P. Simulation of light intensity dependent current characteristics of polymer solar cells. J. Appl. Phys. 95, 2816 (2004).

15. Huynh, W. et al. Charge transport in hybrid nanorod-polymer composite photovoltaic cells. Phys. Rev. B 67, 115326 (2003).

16. Waldauf, C., Scharber, M. C., Schilinsky, P., Hauch, J. A. \& Brabec, C. J. Physics of organic bulk heterojunction devices for photovoltaic applications. J. Appl. Phys. 99, 104503 (2006).

17. Shockley, W. \& Read, W. Statistics of the recombinations of holes and electrons. Phys. Rev. 87, 835-842 (1952).

18. Sinton, R. A. \& Swanson, R. M. Recombination in highly injected silicon. IEEE Trans. Electron Devices 34, 1380-1389 (1987).

19. Ahme, H., Lee, M., Im, C. \& Würfel, U. Influence of the acceptor on electrical performance and charge carrier transport in bulk heterojunction solar cells with HXS-1. J. Phys. Chem. C 118, 3386-3392 (2014).

20. Tress, W. et al. Imbalanced mobilities causing S-shaped IV curves in planar heterojunction organic solar cells. Appl. Phys. Lett. 98, 63301 (2011).

21. Synopsis. TCAD Sentaurus: Sentaurus Device User Guide (2013; Release H-2013.03.

22. Stelzl, F. F. \& Würfel, U. Modeling the influence of doping on the performance of bulk heterojunction organic solar cells: one-dimensional effective semiconductor versus two-dimensional donor/acceptor model. Phys. Rev. B 86, 75315 (2012).

23. Seßler, M., Saeed, A., Kohlstädt, M. \& Würfel, U. Systematic variation of the stabilizer to reduce the annealing temperature of sol-gel derived titanium oxide in inverted organic solar cells. Org. Electron. 15, 1407-1412 (2014).

24. Ratcliff, E. L. et al. Investigating the influence of interfacial contact properties on open circuit voltages in organic photovoltaic performance: work function versus selectivity. Adv. Energy Mater. 3, 647-656 (2013).

25. Ratcliff, E. L., Zacher, B. \& Armstrong, N. R. Selective interlayers and contacts in organic photovoltaic cells. J. Phys. Chem. Lett. 2, 1337-1350 (2011).

26. Reinhardt, J., Grein, M., Bühler, C., Schubert, M. \& Würfel, U. Identifying the impact of surface recombination at electrodes in organic solar cells by means of electroluminescence and modeling. Adv. Energy Mater. 4, 1400081 (2014).

27. Albrecht, S. et al. On the efficiency of charge transfer state splitting in polymer:fullerene solar cells. Adv. Mater. 26, 2533-2539 (2014).

28. Etzold, F. et al. The effect of solvent additives on morphology and excited-state dynamics in PCPDTBT:PCBM photovoltaic blends. J. Am. Chem. Soc. 134, 10569-10583 (2012)

29. Shuttle, C. G., Hamilton, R., O’Regan, B. C., Nelson, J. \& Durrant, J. R. Charge-density-based analysis of the current-voltage response of polythiophene/ fullerene photovoltaic devices. Proc. Natl Acad. Sci. USA 107, 16448-16452 (2010).
30. Li, W. et al. Mobility-controlled performance of thick solar cells based on fluorinated copolymers. J. Am. Chem. Soc. 136, 15566-15576 (2014).

31. Abou-Ras, D., Kirchartz, T. \& Rau, U. (eds.) Advanced Characterization Techniques for Thin Film Solar Cells (Wiley-VCH Verlag GmbH \& Co. KGaA, Weinheim, Germany, 2011).

32. Wetzelaer Gert-Jan, A. H., Kuik, M. \& Blom Paul, W. M. Identifying the nature of charge recombination in organic solar cells from charge-transfer state electroluminescence. Adv. Energy Mater. 2, 1232-1237 (2012).

33. Potscavage, W. J., Yoo, S. \& Kippelen, B. Origin of the open-circuit voltage in multilayer heterojunction organic solar cells. Appl. Phys. Lett. 93, 193308 (2008).

34. Wetzelaer, G. A. H., Kuik, M., Lenes, M. \& Blom, P. W. M. Origin of the darkcurrent ideality factor in polymer:fullerene bulk heterojunction solar cells. Appl. Phys. Lett. 99, 153506 (2011).

35. Mandoc, M. M., Kooistra, F. B., Hummelen, J. C., de Boer, B. \& Blom, P. W. M Effect of traps on the performance of bulk heterojunction organic solar cells. Appl. Phys. Lett. 91, 263505 (2007).

36. Kirchartz, T., Deledalle, F., Tuladhar, P. S., Durrant, J. R. \& Nelson, J. On the differences between dark and light ideality factor in polymer:fullerene solar cells. J. Phys. Chem. Lett. 4, 2371-2376 (2013).

37. Deibel, C., Rauh, D. \& Foertig, A. Order of decay of mobile charge carriers in P3HT:PCBM solar cells. Appl. Phys. Lett. 103, 43307 (2013).

38. Deledalle, F., Shakya Tuladhar, P., Nelson, J., Durrant, J. R. \& Kirchartz, T. Understanding the apparent charge density dependence of mobility and lifetime in organic bulk heterojunction solar cells. J. Phys. Chem. C 118, 8837-8842 (2014).

39. Ou, K.-L. et al. Titanium dioxide electron-selective interlayers created by chemical vapor deposition for inverted configuration organic solar cells. J. Mater. Chem. A 1, 6794 (2013).

40. Liao, S. -H., Jhuo, H. -J., Cheng, Y. -S. \& Chen, S. -A. Fullerene derivativedoped zinc oxide nanofilm as the cathode of inverted polymer solar cells with low-bandgap polymer (PTB7-Th) for high performance. Adv. Mater. 25, 4766-4771 (2013).

41. Das, S. \& Alford, T. L. Improved efficiency of P3HT:PCBM solar cells by incorporation of silver oxide interfacial layer. J. Appl. Phys. 116, 44905 (2014).

42. Würfel, U., Peters, M. \& Hinsch, A. Detailed experimental and theoretical investigation of the electron transport in a dye solar cell by means of a threeelectrode configuration. J. Phys. Chem. C 112, 1711-1720 (2008).

43. Würfel, U., Wagner, J. \& Hinsch, A. Spatial electron distribution and its origin in the nanoporous TiO2 network of a dye solar cell. J. Phys. Chem. B 109, 20444-20448 (2005).

44. Kniepert, J., Schubert, M., Blakesley, J. C. \& Neher, D. Photogeneration and recombination in $\mathrm{P} 3 \mathrm{HT} / \mathrm{PCBM}$ solar cells probed by time-delayed collection field experiments. J. Phys. Chem. Lett. 2, 700-705 (2011).

45. Albrecht, S. et al. Fluorinated copolymer PCPDTBT with enhanced opencircuit voltage and reduced recombination for highly efficient polymer solar cells. J. Am. Chem. Soc. 134, 14932-14944 (2012).

46. Mihailetchi, V. D., Xie, H. X., de Boer, B., Koster, L. J. A. \& Blom, P. W. M. Charge transport and photocurrent generation in poly(3-hexylthiophene): methanofullerene bulk-heterojunction solar cells. Adv. Funct. Mater. 16, 699-708 (2006)

47. Hamilton, R. et al. Recombination in annealed and nonannealed polythiophene/fullerene solar cells: transient photovoltage studies versus numerical modeling. J. Phys. Chem. Lett. 1, 1432-1436 (2010).

48. Rauh, D., Deibel, C. \& Dyakonov, V. Charge density dependent nongeminate recombination in organic bulk heterojunction solar cells. Adv. Funct. Mater. 22 3371-3377 (2012).

49. Scharber, M. C. et al. Design rules for donors in bulk-heterojunction solar cells-towards 10\% energy-conversion efficiency. Adv. Mater. 18, 789-794 (2006).

50. Tvingstedt, K. et al. Electroluminescence from charge transfer states in polymer solar cells. J. Am. Chem. Soc. 131, 11819-11824 (2009).

51. Vandewal, K., Tvingstedt, K., Gadisa, A., Inganäs, O. \& Manca, J. V. Relating the open-circuit voltage to interface molecular properties of donor:acceptor bulk heterojunction solar cells. Phys. Rev. B 81, 125204 (2010).

52. Proctor, C. M., Love, J. A. \& Nguyen, T. -Q. Mobility guidelines for high fill factor solution-processed small molecule solar cells. Adv. Mater. 26, 5957-5961 (2014).

53. Liu, Y. et al. Aggregation and morphology control enables multiple cases of high-efficiency polymer solar cells. Nat. Commun. 5, 5293 (2014).

54. Dennler, G., Scharber, M. C. \& Brabec, C. J. Polymer-Fullerene bulkheterojunction solar cells. Adv. Mater. 21, 1323-1338 (2009).

\section{Acknowledgements}

U.W. and A.S. thank the Deutsche Forschungsgemeinschaft DFG for financial support in the framework of the priority programme SPP1355 ('Elementary processes of organic 
photovoltaics'). D.N. and S.A. further acknowledge funding from the BMBF within the PV comB project (FKZ 03IS2151D).

\section{Author contributions}

U.W. and A.S. carried out the simulations. D.N. and U.W. developed and evaluated the analytical approach. U.W., A.S. and D.N. wrote the paper, with input from S.A.

\section{Additional information}

Supplementary Information accompanies this paper at http://www.nature.com/ naturecommunications

Competing financial interests: The authors declare no competing financial interests.
Reprints and permission information is available online at http://npg.nature.com/ reprintsandpermissions/

How to cite this article: Würfel, U. et al. Impact of charge transport on current-voltage characteristics and power-conversion efficiency of organic solar cells. Nat. Commun. 6:6951 doi: 10.1038/ncomms7951 (2015).

(c) (i) This work is licensed under a Creative Commons Attribution 4.0 International License. The images or other third party material in this article are included in the article's Creative Commons license, unless indicated otherwise in the credit line; if the material is not included under the Creative Commons license, users will need to obtain permission from the license holder to reproduce the material. To view a copy of this license, visit http://creativecommons.org/licenses/by/4.0/ 